\title{
The progenitor of a type Ia supernova with a short delay time?
}

\author{
S. Mereghetti ${ }^{1}$, N. La Palombara ${ }^{1}$, A. Tiengo ${ }^{1,2}$, P. Esposito ${ }^{3}$, \\ L. Stella ${ }^{4}$, and G.L. Israel ${ }^{4}$ \\ ${ }^{1}$ INAF, IASF-Milano, v. E.Bassini 15, I-20133 Milano, Italy, email: \\ sandro@iasf-milano.inaf .it \\ ${ }^{2}$ IUSS, v.le Lungo Ticino Sforza 56, I-27100 Pavia, Italy \\ ${ }^{3}$ INAF - Oss. Astron. di Cagliari, loc. Poggio dei Pini, strada 54, I-09012 Capoterra, Italy \\ ${ }^{4}$ INAF - Osservatorio Astronomico di Roma, v. Frascati 33, I-00040 Monteporzio Catone, Italy
}

\begin{abstract}
.
HD 49798/RX J0648.0-4418 is the only known X-ray binary composed of a hot subdwarf and a massive white dwarf $\left(\mathrm{M}=1.28 \pm 0.05 \quad M_{\odot}\right)$. This system, with an orbital period of 1.55 days, is the outcome of a common envelope evolution, most likely of a pair of stars with initial masses of 8-10 $M_{\odot}$. When the hot subdwarf, currently in a He-burning phase, will expand again and fill its Roche-lobe, the enhanced mass transfer can rapidly bring the already massive white dwarf above the Chandrasekhar limit. The possible final fate, either a Type Ia supernova explosion or an accretion induced collapse, is particularly interesting in view of the high rotational velocity of this star, which has the shortest spin period $(13 \mathrm{~s})$ observed in a white dwarf.
\end{abstract}

Keywords. Stars: subdwarfs, white dwarfs, individual (HD 49798); X-rays: binaries, individual (RX J0648.0-4418).

\section{Introduction}

The binary HD 49798/RXJ0648.0-4418 has been proposed by the organizers of this conference as one of the "mystery object of the day". Indeed, the peculiar properties of the bright star HD 49798 attracted the attention of many astronomers since the time of its first observations in the sixties. The discovery of pulsed soft X-rays with ROSAT in 1996 renewed interest in this binary, while our more recent results showed its relevance for the topics discussed in this IAU Symposium.

Here, after briefly reviewing how the main mystery, i.e. the nature of the unseen companion of HD 49798, was finally solved thanks to X-ray observations, we discuss a few other puzzling issues still to be clarified to fully understand this unique binary system.

\section{The peculiar properties of HD 49798}

The first spectroscopic observations of this bright blue star $(B=8, B-V=-0.27)$ showed a dominance of $\mathrm{He}$ and $\mathrm{N}$ lines and radial velocity variations, pointing to a binary nature. Jaschek \& Jaschek (1963) included HD 49798 in the (then small) group of early type subdwarfs and, pointing out the peculiarities of its spectrum, concluded their paper with the sentence "The orbit of this object would obviously be very desirable". A few years later the orbital period (1.5477 days) and mass function were derived by Thackeray (1970), who also pointed out the lack of eclipse and/or ellipsoidal variations in the optical light curve. On the basis of these findings he suggested that the companion of HD 49798 could be a white dwarf, but the possibility of a late type main sequence star, outshined in the 
optical/UV spectra by the much brighter emission from the hot and luminous subdwarf $\left(\mathrm{L} \sim 10^{4} L_{\odot}\right)$, could not be excluded. In the following years several studies concentrated on a detailed modeling of the star's atmosphere (e.g., Richter(1971), Dufton(1972)). HD 49798 was classified as a subdwarf of spectral type O6, with an effective temperature $\mathrm{T}_{\text {eff }}=47500 \pm 2000 \mathrm{~K}$, and a surface gravity $\log g=4.25 \pm 0.2$ (Kudritzki \& Simon 1978). The overabundance of Helium, equalling $\mathrm{H}$ in number, was confirmed (mass fractions of $\mathrm{X}_{H}=0.29$ and $\mathrm{X}_{H e}=0.78$ ). The low $\mathrm{H}$ abundance suggests that HD 49798 is the stripped core of an initially much more massive and larger star. Also the high abundance of $\mathrm{N}$ and low abundance of $\mathrm{C}$ and $\mathrm{O}$ confirm that its present surface layers once belonged to the outer part of the hydrogen-burning core of a massive star, consistent with an evolution involving a common envelope phase. The optical mass function could be measured with great precision by Stickland \& Lloyd (1994) $\left(\mathrm{f}_{O P T}=0.263 \pm 0.004 M_{\odot}\right)$, but all the attempts to reveal the invisible companion of this single-lined spectroscopic binary were unsuccessful.

\section{X-rays reveal the nature of the companion star}

The mystery was partially solved in 1996, thanks to the ROSAT discovery of periodic pulsations at $13 \mathrm{~s}$ in the soft X-ray (0.1-2 keV) flux (Israel et al. 1997). This clearly pointed to the presence of a collapsed object, but it was impossible to distinguish between a neutron star or a white dwarf. The very soft spectrum was well fit with a blackbody plus power-law model, but with a poorly constrained luminosity in the range from $\sim 10^{32}$ erg $\mathrm{s}^{-1}$ up to more than $10^{35} \mathrm{erg} \mathrm{s}^{-1}$ (for the well known distance of $650 \mathrm{pc}$ ), compatible with both possibilities. The X-ray emission from HD 49798 is too soft and faint for satellites like BeppoSAX, ASCA or RossiXTE, so we had to wait for XMM-Newton in order to make further progress.

After four short observations done in 2002 which added little information (Tiengo et al. 2004), we managed to obtain a long pointing of HD 49798 in May 2008, strategically scheduled at the time of conjunctiont. Our main objectives were to exploit the regular X-ray periodicity, which makes this system equivalent to a double spectroscopic binary, to constrain the masses of the two stars, and to get a better estimate of the source spectral parameters and luminosity. The measurement of the X-ray pulse delays induced by the orbital motion, and the discovery of an X-ray eclipse lasting $\sim 1.3$ hours, allowed us to derive the X-ray mass function as well as the system's inclination $\left(79^{\circ}<i<84^{\circ}\right)$. This information, coupled to the already known optical mass function, gives the masses of the two stars: $\mathrm{M}_{s d}=1.50 \pm 0.05 M_{\odot}$ for the subdwarf and $\mathrm{M}_{W D}=1.28 \pm 0.05 M_{\odot}$ for its companion (Mereghetti et al. 2009), Furthermore, the high quality spectrum obtained with the $X M M$-Newton EPIC instrument showed that the total luminosity is only $\sim 10^{32}$

erg $\mathrm{s}^{-1}$, much smaller than that expected from a neutron star accreting in the stellar wind of HD 49798.

\section{More mysteries ?}

While the mysterious companion of HD 49798 has finally been unveiled by X-ray observations, this binary certainly deserves further study. It stands out in the zoo of accreting X-ray binaries for being the only one composed of a white dwarf and a subdwarf. Furthermore, the white dwarf, besides being one of the most massive white dwarfs with a dynamical mass measurement, is the one with the shortest spin period $(\mathrm{P}=13 \mathrm{~s})$. These

$\dagger$ The phase of the expected eclipse, that was never covered in previous X-ray observations. 
properties make HD 49798/RX J0648.0-4418 particularly attractive in the context of type Ia $\mathrm{SNe}$ progenitors.

\subsection{Origin of the fast rotation}

Why is the white dwarf rotating so rapidly? Was it born with a short period or this is the result of spin-up caused by disk accretion, as in recycled millisecond pulsars?

The radius of HD $49798\left(1.45 \pm 0.25 \quad R_{\odot}\right)$ is much smaller than that of its Rochelobe. On the other hand this is one of the few O-type subdwarfs with evidence for a relatively strong stellar wind, with a mass loss of $\sim 3 \times 10^{-9} \quad M_{\odot} \mathrm{yr}^{-1}$ (Hamann 2010), Thus the white dwarf is accreting through capture of the subdwarf's wind, and there is no evidence for an accretion disk. If significant spin-up occurred, this must have been either during the common envelope phase, which seems unlikely owing to its short duration and complicated dynamics of the envelope mass, or before it, possibly when the expanding HD 49798 was close to fill its Roche-lobe. Note that it is relatively easy to spin-up a weakly magnetized white dwarf, due to the high specific angular momentum carried by the accreting mass if the accretion disk extends down to the star's surface (Livio \& Pringle 1998). If the value of $\mathrm{P}=13.2 \mathrm{~s}$ observed now has remained close to the equilibrium period, we can relate the accretion rate during the spin-up phase, $\dot{M}$, to the strength of the magnetic field: $\dot{M} \sim 3 \times 10^{-10} \mu_{30}^{2} M_{\odot} \mathrm{yr}^{-1}$, where $\mu_{30}$ is the magnetic dipole moment in units of $10^{30} \mathrm{G} \mathrm{cm}^{3}$. If indeed the magnetic field of RX J0648.0-4418 is as small as discussed below, one wonders why the white dwarf is not rotating even faster than observed. In any case, independent of its origin, the high rotational velocity might have important consequences for the future evolution of this white dwarf.

\subsection{Properties of the X-ray emission and magnetic field intensity}

The X-ray emission of RX J0648.0-4418 consists of a soft blackbody component, with $\mathrm{kT}=40 \mathrm{eV}$ and a strong nearly sinusoidal modulation (pulsed fraction 56\%), plus a harder component dominating above $\sim 1 \mathrm{keV}$. The latter can be fit equally well by a power law with photon index $\Gamma=1.6$ or by a thermal bremsstrahlung with $\mathrm{kT}=8 \mathrm{keV}$, and shows a double peaked pulse profile (Mereghetti et al. 2011), Such characteristics are quite similar to those of cataclysmic variables of the polar and intermediate polar class, despite these systems are very different for what concerns their mass donor stars. These similarities suggest that that the X-ray emission properties depend mainly on the physical conditions near the white dwarf, with little influence of the large scale accretion scenario (wind accretion in RX J0648.0-4418 wrt Roche lobe overflow in cataclysmic variables).

A condition for accretion onto the white dwarf surface is that the magnetospheric radius, defined by balancing the magnetic pressure and the ram pressure of the accreting matter, be smaller than the corotation radius $\mathrm{R}_{C}=\left(\mathrm{G} \mathrm{M}_{W D} \mathrm{P}^{2} / 4 \pi^{2}\right)^{1 / 3}$. This allows us to estimate an upper limit of $2 \times 10^{29} \mathrm{G} \mathrm{cm}^{3}$ on the magnetic moment of RX J0648.04418. An even stronger limit of $\mu<3 \times 10^{28} \mathrm{G} \mathrm{cm}^{3}$ is obtained assuming that accretion takes place in the sub-sonic propeller regime, in which $\mathrm{R}_{M}<\mathrm{R}_{C}$, but the matter is too hot to accrete (Davies et al. 1979). These limits correspond to fields of the order of only a few $\mathrm{kG}$ at the white dwarf's surface, much smaller than those of cataclysmic variables. With such a small field it is probably difficult to channel the accreting plasma. On the other hand the large pulsed fraction $(\sim 60 \%)$ observed below $1 \mathrm{keV}$ requires some form of non-isotropic emission. One possibility is that the field has a non-dipolar geometry, given that the above limits refer only to the dipolar component. 


\subsection{Future evolution}

At the end of the current He-burning phase, HD 49798 will expand again and transfer Herich matter through Roche-lobe overflow during a semi-detached phase (Iben \& Tutukov 1994). but the fraction of mass that is retained on the white dwarf is rather uncertain. Recent computations, performed assuming the mass accumulation efficiency that takes into account the wind mass loss triggered by the He-shell flashes (Kato \& Hachisu 2004), indicate that a mass of $1.4 M_{\odot}$ can be reached after only a few $10^{4}$ years (Wang \& Han 2010), However, there are other critical factors which influence the final fate of the white dwarf, such as, e.g., its composition and rotational velocity.

If RX J0648.0-4418 is a CO white dwarf, it could be the progenitor of an over-luminous type Ia supernova, since the fast rotation can increase the mass stability limit above the value for non-rotating stars. Massive white dwarfs are expected to have an ONe composition, but again the high spin might play a role here, since it can lead to the formation of CO white dwarfs even for high masses (Dominguez et al. 1996), Being the result of the evolution of relatively massive stars ( $\left.\sim 8-9 \quad M_{\odot}\right)$, HD 49798/RX J0648.04418 would seem a type Ia supernova progenitor with a short delay time. However, the delay time might be considerably longer if the explosion has to await that the white dwarf spins down (Di Stefano et al. 2011)

Alternatively, if RX J0648.0-4418 is an ONe white dwarf, an accretion induced collapse might occur leading to the formation of a neutron star. The high spin rate and low magnetic field make this white dwarf an ideal progenitor of a millisecond pulsar. The evolution of systems like HD 49798/RX J0648.0-4418 could be a promising scenario for the direct formation of millisecond pulsars, i.e. one not involving the recycling of old pulsars in accreting low mass X-ray binaries.

\section{Acknowledgements}

We acknowledge financial contribution from the agreements ASI-INAF I/032/10/0. PE acknowledges financial support through grant Sardegna FSE 2007-2013, L.R. 7/2007.

\section{References}

Davies, R. E., Fabian, A. C., \& Pringle, J. E. 1979, MNRAS, 186, 779

Di Stefano, R., Voss, R., \& Claeys, J. S. W. 2011, ApJ, 738, L1

Dominguez, I., Straniero, O., Tornambe, A., \& Isern, J. 1996, ApJ, 472, 783

Dufton, P. L. 1972, MNRAS, 159, 79

Hamann, W. 2010, Ap $6 S S, 119$

Hamann, W., Gruschinske, J., Kudritzki, R. P., \& Simon, K. P. 1981, A\&A, 104, 249

Heber, U. 2009, ARA\&A, 47, 211

Iben, Jr., I. \& Tutukov, A. V. 1985, ApJS, 58, 661

Iben, I. J. \& Tutukov, A. V. 1993, ApJ, 418, 343

Iben, I. J. \& Tutukov, A. V. 1994, ApJ, 431, 264

Israel, G. L., Stella, L., Angelini, L., et al. 1997, ApJ, 474, L53

Jaschek, M. \& Jaschek, C. 1963, PASP, 75, 365

Kato, M. \& Hachisu, I. 2004, ApJ, 613, L129

Kudritzki, R. P. \& Simon, K. P. 1978, A\&A, 70, 653

Livio, M., \& Pringle, J. E. 1998, ApJ, 505, 339

Mereghetti, S., Tiengo, A., Esposito, P., et al. 2009, Science, 325, 1222

Mereghetti, S., La Palombara, N., Tiengo, A., et al. 2011, ApJ, 737, 51

Richter, D. 1971, A\& A, 14, 415

Stickland, D. J. \& Lloyd, C. 1994, The Observatory, 114, 41

Thackeray, A. D. 1970, MNRAS, 150, 215

Tiengo, A., Mereghetti, S., Israel, G. L., \& Stella, L. 2004, Nucl. Phys. B Proc. Suppl., 132, 705

Wang, B. \& Han, Z. 2010, Research in Astronomy and Astrophysics, 10, 681 